\title{
Efficacy and Safety of Galcanezumab for the Prevention of Episodic Migraine: Results of the EVOLVE-2 Phase 3 Randomized Controlled Clinical Trial
}

Vladimir Skljarevski, MD ${ }^{1}$, Manjit Matharu, $\mathrm{MD}, \mathrm{PhD}^{2}$, Brian A. Millen, $\mathrm{PhD}^{1}$, Michael H. Ossipov, $\mathrm{PhD}^{3}$, Byung-Kun Kim, MD, $\mathrm{PhD}^{4}$, Jyun Yan Yang, $\mathrm{MD}^{1}$

${ }^{1}$ Lilly Research Laboratories, Lilly Corporate Center, Indianapolis, IN, USA

${ }^{2}$ National Hospital for Neurology and Neurosurgery, London, United Kingdom

${ }^{3}$ Clinical Division, Syneos Health Raleigh, NC, USA

${ }^{4}$ Eulji General Hospital, Seoul, Republic of Korea

\section{Corresponding Author:}

Vladimir Skljarevski, MD

Eli Lilly and Company

Lilly Corporate Center

Indianapolis, IN 46285 United States

Work Phone: 317-651-2173

E-Mail: skljarevski_vladimir@lilly.com

Trial Registration: NCT02614196

Key words: Episodic migraine; headache; CGRP; humanized monoclonal antibody; galcanezumab; LY2951742, MSQ, MIDAS

Word Count: 3443 (Limit is 3500)

References: 23

Tables: 4

Figures: 9 


\begin{abstract}
Introduction: Galcanezumab, a humanized monoclonal antibody binding calcitonin gene-related peptide, in development for migraine prevention.

Methods: In this global, double-blind, 6-month study of patients with episodic migraine, the intent to treat population included 915 patients randomized to monthly subcutaneous injections of galcanezumab $120 \mathrm{mg}(\mathrm{N}=231)$ or $240 \mathrm{mg}(\mathrm{N}=223)$ or placebo $(\mathrm{N}=461)$. Primary endpoint was overall mean change from baseline in monthly migraine headache days. Key secondary endpoints were $\geq 50 \%, \geq 75 \%$, and $100 \%$ response rates; monthly migraine headache days with acute migraine medication use; Patient Global Impression of Severity rating; and the Role Function-Restrictive of the Migraine-Specific Quality of Life questionnaire.
\end{abstract}

Results: Mean monthly migraine headache days were reduced by 4.3 and 4.2 days by galcanezumab 120 and $240 \mathrm{mg}$, respectively, and 2.3 days by placebo. Both doses were also superior to placebo for all key secondary endpoints. Injection site pain was the most common treatment-emergent adverse event, reported at similar rates in placebo and galcanezumab treatment groups. Both galcanezumab doses had significantly more injection site reactions and injection site pruritus, and the $240 \mathrm{mg}$ group had significantly more injection site erythema relative to placebo.

Conclusions: Galcanezumab 120 or 240 mg given once monthly was efficacious, safe, and well tolerated.

Study identification: EVOLVE-2; NCT02614196;

https://clinicaltrials.gov/ct2/show/NCT02614196?term=LY2951742\%2C+cgah\&rank=1 


\section{Introduction}

The worldwide prevalence of migraine and its associated socioeconomic cost are well recognized (1-3). Despite its heavy burden, migraine is commonly inadequately treated (4,5). For example, the American Migraine Prevalence and Prevention study revealed that $54 \%$ of patients with migraine needed bed rest or reported severe impairment, and 39\% reported some impairment (6). Moreover, although prevention treatment was indicated in $39 \%$ of patients with migraine, only $13 \%$ received it (6). In addition, up to $68 \%$ of patients who use preventive medications stop doing so within 6 months because of insufficient benefit, dissatisfaction with the drug, or poor tolerability (7-9). There is a significant need for new treatment options with improved efficacy and tolerability.

Over the past 3 decades, clinical investigations have pointed to the critical role of calcitonin gene-related peptide (CGRP) in migraine pathophysiology (10-13). This neuropeptide is expressed throughout the peripheral and central nervous systems and is prominent within the trigeminovascular system (12). It has been implicated in sensory neuromodulation, vasodilation, and mediation of neurogenic inflammation (12-14). Several studies demonstrated that migraine attacks are accompanied by elevated blood levels of CGRP, and the infusion of CGRP induces headache in individuals with migraine $(10,11,15-17)$. Selective antagonists to the CGRP receptor were efficacious in clinical trials for acute migraine (12).

Galcanezumab is a humanized monoclonal antibody that binds CGRP and prevents its biological activity without blocking the CGRP receptor. Two Phase 2 clinical trials have shown that galcanezumab, given in monthly doses ranging from 120 to $300 \mathrm{mg}$, was safe and efficacious in migraine prevention $(18,19)$. In the current manuscript, we hypothesized that at least one dose of galcanezumab was superior to placebo over a 6-month dosing regimen. Accordingly, we provide the results of a large, global, Phase 3 trial of galcanezumab that confirms its efficacy and safety in patients with episodic migraine. 


\section{Methods}

Study Design

EVOLVE-2 (NCT02614196) was a Phase 3, multi-center, placebo-controlled, double-blind, randomized clinical trial analyzing the efficacy and safety of two dosing regimens of galcanezumab in patients with episodic migraine. This study was conducted between January 2016 and March 2017 at 109 study sites in the United States, United Kingdom, Netherlands, Spain, Czech Republic, Germany, Argentina, Israel, Korea, Taiwan, and Mexico. Of these sites, 49 (45\%) were in the US. The study was composed of 4 study periods (Figure 1). Study Period I consisted of medical examination and washout of migraine preventive medications for at least 30 days (4 months for onabotulinumtoxin A). Study Period II was a 1-month baseline period that was used to establish the baseline number of migraine headache days (MHDs). Study Period III was a 6-month double-blind treatment phase. During the post-baseline periods, patients were allowed to take acute migraine medications as needed, with the exception of medications containing opioids or barbiturates, which were limited to no more than 3 times per month. Study Period IV was a 4month post-treatment (washout) period and is not a topic of this manuscript. Key elements of the protocol are available at https://clinicaltrials.gov/ct2/show/NCT02614196?term=LY2951742\%2C+cgah\&rank=1.

A minimum of 825 patients were planned for this study, randomized in a 2:1:1 ratio of placebo to galcanezumab $120 \mathrm{mg} / \mathrm{month}$ or $240 \mathrm{mg} / \mathrm{month}$ (target of 206 patients). It was estimated based on Monte Carlo simulations that this sample size would provide approximately $95 \%$ power that at least 1 dose level of galcanezumab would separate from placebo on the primary endpoint, at an overall one-sided 0.025 significance level, assuming a standardized treatment effect size of 0.33 and a $26 \%$ discontinuation rate.

\section{Inclusion Criteria:}

To be enrolled in the study, patients had to be between the ages of 18 and 65 years (inclusive) and have a diagnosis of migraine with or without aura (1.1 and 1.2), as defined by International Headache Society (IHS) International Classification of Headache Disorders, 3rd edition, (ICHD-3) beta version (3). Patients were enrolled into the study by being approached by study staff, or through referral or advertising. 
Included patients had a history of migraine for at least 1 year prior to enrollment and with migraine onset prior to age 50 years. Patients also had to have 4 to 14 MHDs (including probable MHD), at least 2 migraine attacks during the baseline period, and an $80 \%$ compliance rate in using the electronic diary (ePRO). Finally, patients had to agree to use an acceptable method of birth control during the study and for at least 5 months after receiving the last dose. The study protocol was reviewed and approved by the appropriate institutional review board for each of the study sites, and the study was conducted according to Good Clinical Practice and the Declaration of Helsinki guidelines. Patients provided written informed consent before undergoing study procedures. Investigators at each study site evaluated and confirmed eligibility, obtained consent and enrolled the patients.

\section{Exclusion Criteria}

Patients were excluded if they had failed treatment with 3 or more migraine prevention drugs from different classes (level A or B evidence per American Academy of Neurology guidelines for episodic migraine prevention) (20) or if they were using opioids or barbiturates more than twice per month. Other conditions leading to exclusion were participation in another clinical trial within the past 30 days, prior exposure to galcanezumab or any another CGRP antibody, taking any therapeutic antibody in the past 12 months, known hypersensitivity to multiple drugs, or presence of any medical or psychiatric illness that would preclude study participation.

\section{Randomization and Blinding}

Randomization to double-blind treatment was performed at Visit 3. Patients were assigned in a doubleblind manner by a computer-generated randomization sequence using an interactive web-response system (IWRS) to placebo or galcanezumab 120 or $240 \mathrm{mg}$ (2:1:1 ratio) administered by subcutaneous injection once monthly for 6 months. Patients randomized to the 120-mg dose received a loading dose of $240 \mathrm{mg}$ (2 injections of $120 \mathrm{mg}$ each) at the first dosing visit. To achieve balance among groups, randomization was stratified by country and migraine frequency ( $<8 \mathrm{vs.} \geq 8 \mathrm{MHDs} / \mathrm{month})$ at baseline. All injections were 
administered by the investigator sites' trained personnel. In order to preserve blinding, galcanezumab and matching placebo (ie; excipients only) were supplied as 1-mL, single-dose, prefilled, disposable manual syringes with study-specific labels. The syringes were visibly indistinguishable from each other. Patients in all groups received two injections at each dosing visit to preserve blinding throughout the study.

\section{Outcome Measures}

Efficacy analyses were performed in the intent-to-treat (ITT) population, where each randomized patient had received at least one dose of galcanezumab or placebo. The primary objective was to assess whether at least one of the studied dosing regimens of galcanezumab was superior to placebo in the prevention of migraine headache in patients with episodic migraine. The primary analysis evaluated the efficacy of each dosing regimens of galcanezumab compared with placebo on the overall mean change from baseline in the number of monthly MHDs during the 6-month treatment phase, based on the ePRO data. The key secondary outcome measures, analyzed with control for multiple testing, were the following:

- The mean proportion of patients with reduction from baseline of $\geq 50 \%, \geq 75 \%$, and $100 \%$ in monthly MHDs during the 6-month double-blind treatment phase. Response rate was defined as the percentage of patients meeting predefined thresholds (ie; $\geq 50 \%, \geq 75 \%$, and $100 \%$ ) in the reduction from baseline in the number of MHDs for each month, and the overall percentage of patients meeting these thresholds averaged over months 1 through 6 were analyzed.

- The mean change from baseline in the Role Function-Restrictive (R-FR) domain score of the Migraine-Specific Quality of Life Questionnaire version 2.1 (MSQ v2.1), as an average of Months 4 to 6.

- The overall mean change from baseline in the number of MHDs with acute migraine medication use during the 6-month double-blind treatment phase. 
- The mean change from baseline in the Patient Global Impression of Severity (PGI-S) rating (average of Months 4 to 6).

A secondary, but non-key, outcome measure that is included in the current manuscript is the Migraine Disability Assessment (MIDAS) total score at Month 6.

\section{Safety and Tolerability}

The safety population consisted of all randomized patients who received at least one dose of galcanezumab or placebo. Adverse events were coded by Medical Dictionary for Regulatory Activities (version 19.1). The safety parameters reported in the present manuscript are the treatment-emergent adverse events (TEAEs), serious adverse events (SAEs), deaths, discontinuations due to adverse events, discontinuation rates, vital signs, and body weight. The topic of immunogenicity is also briefly discussed. A comprehensive safety report is in preparation as a separate manuscript.

\section{Assessments}

The ePRO daily diary was used to record headache and other migraine symptoms. Based on the diary data, using an automated algorithm, the days were categorized as MHDs (including probable MHD). A probable migraine is defined as a headache with or without aura and lasting $\geq 30$ minutes but missing one of the migraine features in the ICHD-3 beta criteria (3). The monthly number of MHDs with acute migraine medication use was obtained through the ePRO diary, and the PGI-S, MQS, and MIDAS assessments were performed at the study site at every monthly visit for PGI-S and MSQ and on Months 3 and 6 for MIDAS with the use of a slate device.

\section{Statistical Analyses}

The changes from baseline to each scheduled postbaseline measurement for continuous efficacy measures were estimated for each treatment from mixed effect model repeat measurements (MMRM) analyses. This model included the fixed, categorical effects of treatment, pooled country, month, and treatment-by- 
month interaction, as well as the continuous, fixed covariates of baseline value and baseline-by-month interaction. The primary endpoint of overall mean change in monthly MHDs was estimated as the main effect of treatment (each galcanezumab dose group and placebo) during the 6-month treatment phase. This provided the average treatment effect across the 6-month double-blind treatment phase.

When the objective of the continuous secondary efficacy measures was to assess overall mean change during the 6-month treatment phase, the endpoint for comparing galcanezumab with placebo was the main effect of treatment from the MMRM analysis across Months 1 to 6 . Change from baseline of continuous variables with repeated measures included the change from baseline for MHD with acute medication use for Months 1 to 6, as well as MSQ R-FR and PGI-S for Months 4 to 6. The categorical longitudinal efficacy measures of $\geq 50 \%, \geq 75 \%$, and $100 \%$ reduction from baseline in the number of monthly MHD from Months 1 to 6 were analyzed with a categorical, pseudo-likelihood-based repeated measures analysis. A superchain (multiple testing) procedure was used to ensure strong control of the familywise type I error rate for the primary and key secondary endpoints (Figure 2) (21). According to the specified procedure, testing of key secondary endpoints was to commence in a sequential fashion following testing of the primary endpoint. The superchain procedure provides appropriate adjustment for multiple testing across the two dose levels and all primary and key secondary endpoints. Categorical safety measures were analyzed using the Fisher's exact test. All statistical analyses were performed with the use of SAS Enterprise Guide 7.1 (SAS Institute, Cary, NC).

\section{Results}

\section{Patient Disposition and Baseline Characteristics}

A total of 1696 patients entered study screening, and 922 were randomized (Figure 3). The most common reason for patients failing the screening phase was not having the required number of MHDs. A total of 915 patients received at least one dose of galcanezumab or placebo and comprised the ITT population, and 785 patients $(85.8 \%)$ completed the double-blind treatment phase of the study. Overall, a total of 129 (14.1\%) patients discontinued during Study Period III. The most common reason for discontinuation was 
the patient's decision to withdraw, accounting for 64 (7\%) patients. Adverse events (AEs) accounted for the discontinuation of $8(1.7 \%)$ placebo patients, $5(2.2 \%)$ galcanezumab $120 \mathrm{mg}$ patients, and $9(4.0 \%)$ galcanezumab $240 \mathrm{mg}$ patients and were not significantly different among the groups. In addition, 10 (2.2\%) placebo patients, 7 (3.0\%) galcanezumab $120 \mathrm{mg}$ patients, and $0(0 \%)$ galcanezumab $240 \mathrm{mg}$ patients were lost to follow-up.

Mean age (standard deviation [SD]) of the study population was 41.9 (11.1) years, and the population was largely female (85.4\%) and white (70.3\%), and North American (48.7\%) or European (26.3\%). At baseline, approximately $66.9 \%$ of patients had 8 or more MHDs per month. The majority $(65.5 \%)$ of the patients had prior experience with migraine preventive treatments, and $14.3 \%$ of them had previously failed 2 or more preventive medications. The baseline characteristics for each treatment group are summarized in Table 1.

\section{Efficacy and Measures of Functioning}

Galcanezumab at both doses met the primary and all key secondary endpoints (Table 2). A total of 896 of the 915 ITT patients were included in the efficacy analyses. Nineteen patients were excluded because they did not meet the requirement of having at least one non-missing post-baseline monthly MHD. The least squares (LS) mean change from baseline in MHDs over the 6-month study period for galcanezumab 120 and $240 \mathrm{mg}$ were significantly $(\mathrm{p}<.001)$ reduced relative to placebo (Figure 4A). These reductions represent changes of $-2.02( \pm 0.27)$ and $-1.90( \pm 0.27)$ monthly MHDs, relative to placebo, for galcanezumab 120 and $240 \mathrm{mg}$, respectively. The reduction in monthly MHDs for the galcanezumab 120 and $240 \mathrm{mg}$ groups separated from placebo at a statistically significant level at Month 1 and maintained that separation throughout the treatment phase of the study (Figure 4B).

Significantly $(\mathrm{p}<.001)$ greater reductions in monthly MHDs response rates of $\geq 50 \%$ were observed in $59 \%$ and $57 \%$ of patients in the galcanezumab 120 and $240 \mathrm{mg}$ groups, respectively, when compared to the $36 \%$ in the placebo group (Figure 5). The percentages of patients reporting $\geq 75 \%$ and $100 \%$ response 
rates are provided in Figure 5. Galcanezumab 120 and $240 \mathrm{mg}$ resulted in significant $(\mathrm{p}<.001)$ reductions in MHDs with acute migraine medication use compared to the placebo group (Figure 6).

Treatment with both dosing regimens of galcanezumab was associated with reduced functional impairment due to migraine as measured in the MSQ RF-R. Both galcanezumab 120 and $240 \mathrm{mg}$ significantly ( $p<.001)$ improved the LS means of the MSQ RF-R score averaged over Months 4 through 6 (Figure 7). Both galcanezumab groups showed improvement in patients' global impression of severity of their disease as assessed by PGI-S rating (Figure 8). The LS mean change in PGI-S ratings for the galcanezumab 120 and $240 \mathrm{mg}$ groups were significantly ( $\mathrm{p}=.002$ and $\mathrm{p}=.012$, respectively) greater than in the placebo group. In addition, both treatment groups of galcanezumab significantly $(\mathrm{p}<.001)$ improved total MIDAS scores at Month 6, which was the prespecified time point of interest, compared to placebo group (Figure 9).

\section{Safety and Tolerability}

TEAEs were reported by $147(65.0 \%)$ and 163 (71.5\%) of the patients receiving galcanezumab 120 and $240 \mathrm{mg}$, respectively, and by 287 (62.3\%) placebo patients (Table 3). The proportion of patients reporting a TEAE from the galcanezumab $240 \mathrm{mg}$ group was significantly $(\mathrm{p}<.05)$ greater than that of the placebo group. No deaths were reported in any of the treatment groups, and the percentage of SAEs, which were $1.1 \%, 2.2 \%$, and $3.1 \%$ for placebo, galcanezumab $120 \mathrm{mg}$, and galcanezumab $240 \mathrm{mg}$ groups, respectively, did not differ significantly (Table 3). Twenty SAEs were reported by 17 patients. Five placebo patients had gallbladder polyp, hemorrhoids, migraine, and suicide attempt. Foot fracture, rib fracture, and road traffic accident were all reported by 1 placebo patient. Five patients in the galcanezumab $120 \mathrm{mg}$ group had adenocarcinoma of the cervix, bladder dysfunction, gastritis, pharyngitis bacterial, and rectal polyp; and 7 patients in the galcanezumab $240 \mathrm{mg}$ group reported acute myocardial infarction, cholelithiasis, generalized tonic-clonic seizure, influenza, meniscus injury, and transient ischemic attack. Disorientation and pyrexia were both reported by 1 patient in the galcanezumab $240 \mathrm{mg}$ 
group. None of these SAEs were reported by more than 1 patient. The percentage of patients discontinuing the study because of adverse effects were 1.7\% (8 patients reporting atopic dermatitis, facial pain, fatigue, hypertension, pain in extremity, suicide attempt, syncope, vertigo), $2.2 \%$ (5 patients reporting injection site reaction, adenocarcinoma of the cervix, bronchiectasis, gastritis, pruritic rash), and 4.0\% (9 patients reporting injection site reaction, chest discomfort, hepatic enzyme increased, infection, influenza like illness, nasopharyngitis, skin ulcer) for the placebo, galcanezumab $120 \mathrm{mg}$, and galcanezumab $240 \mathrm{mg}$ groups, respectively, and did not differ significantly among the 3 treatment groups (Table 3). The most commonly reported TEAE among the 3 treatment groups was injection site pain $(9.3 \%, 8.8 \%$, and $8.5 \%$ for the galcanezumab $120 \mathrm{mg}$, galcanezumab $240 \mathrm{mg}$, and placebo groups, respectively), which was not significantly different among the groups. Commonly reported TEAEs that showed significant differences for either or both dosing regimens of galcanezumab compared to placebo were injection site reaction, injection site erythema, injection site pruritus, and injection site swelling (Table 4). No statistically significant differences were found in mean change from baseline for pulse rate, body weight, systolic/diastolic blood pressure, and body temperature between the galcanezumab treatment groups and placebo.

Immunogenicity

At baseline, prior to randomization, anti-drug antibodies (ADAs) were detected in 8.4\%, 8.1\%, and 11.2\% of patients in the placebo, galcanezumab $120 \mathrm{mg}$, and galcanezumab $240 \mathrm{mg}$ groups, respectively. There were $19(8.6 \%), 11(5.1 \%)$, and $2(0.5 \%)$ patients in the galcanezumab $120 \mathrm{mg}$, galcanezumab $240 \mathrm{mg}$, and placebo groups, respectively, with treatment-emergent ADA. The difference between each galcanezumab treatment group and placebo was statistically significant $(\mathrm{p}<.001)$. Among all treatmentemergent ADA+ patients, 29 of them had neutralizing ADAs present. The ADA titers were generally low. Titers ranged from not detectable to a maximum of 1:1280, which occurred in 4 patients. Patients who were ADA+ at any time and who also reported a TEAE had titers ranging from 1:10 to 1:640. Treatmentemergent ADAs or neutralizing ADAs had no impact on either safety or efficacy. 



\section{Discussion}

Treatment with the currently available migraine prevention drugs is associated with a relatively poor tolerability and low rates of patient satisfaction $(8,9)$. Consequently, the prevention of migraine remains an important unmet clinical need. The results of this large Phase 3 randomized clinical trial demonstrate that galcanezumab is well-tolerated and efficacious in preventing migraine headache.

Both dose regimens of galcanezumab met the primary and all key secondary endpoints. Galcanezumab significantly reduced the monthly MHDs relative to placebo within the first month of the trial and the reduction was maintained throughout the 6-month treatment period. Mean reduction in MHD observed in the galcanezumab treatment group translates into an annualized gain of approximately 7 weeks of migraine-free days. In addition, $59 \%$ and $57 \%$ of patients who were treated with galcanezumab 120 or $240 \mathrm{mg}$, respectively, relative to $36 \%$ of those receiving placebo, had response rates of $\geq 50 \%$, a wellestablished benchmark indicating a clinically meaningful result (22). Similarly, $\geq 75 \%$ and $100 \%$ response rates were also statistically significantly greater in both glacanezumab treatment groups relative to placebo. Treatment with both dose regimens of galcanezumab was also associated with a significant decrease in the use of acute migraine medications. This is an important consideration because their overuse may lead to the development of medication overuse headache $(3,10)$. Galcanezumab was also found to be superior to placebo in reducing the migraine-related impairment in functioning, as indicated by the significant improvement relative to placebo, as measured by the MSQ R-FR and the MIDAS total scores. The patients' global impression of severity of disease as determined by the PGI-S scale was also significantly improved relative to placebo by both dose regimens of galcanezumab. Taken together, the results of this global clinical trial indicate that (1) treatment with galcanezumab demonstrates a clinically meaningful level of efficacy in preventing migraine headaches in patients with episodic migraine and (2) replicate the findings of the identically designed North American study (EVOLVE-1) (23).

Injection site pain, the most common TEAE, was reported by approximately $9 \%$ of patients, regardless of whether treated with galcanezumab or placebo. TEAEs significantly more commonly reported by the 
galcanezumab-treated patients, relative to those treated with placebo, were all injection site related: injection site reaction, injection site pruritus, injection site swelling, and injection site erythema. Most of these TEAEs were reported to be mild to moderate. No reported serious TEAEs were related to injection site, and only 4 patients receiving galcanezumab discontinued the study due to injection site reaction. Discontinuations due to TEAEs were low, as was the rate of SAEs, and they did not differ among the treatment groups. Together, these results show that galcanezumab is well tolerated, with the small number of patients experiencing relatively minor injection-site related adverse effects.

Galcanezumab presents a promising investigational drug for migraine prevention. By the end of the 6month treatment period, more patients receiving placebo than those receiving galcanezumab discontinued from the study for any reason. Similarly, the number of placebo-treated patients who discontinued the study due to lack of efficacy was 3 times as high as the corresponding number of the galcanezumabtreated patients. These results are especially encouraging when one considers that as many as two-thirds of patients using currently available preventive therapies discontinue their use because of poor tolerability or lack of efficacy over a 6-month period (7-9). Notably, this study also included patients who had tried and failed several preventive treatments from up to two different classes.

Limitations of the study include certain restrictions in the inclusion criteria that may limit the generalizability of the results. Patients with serious medical or psychiatric conditions, high body mass index, substantial opioid use, and high risk for major cardiovascular events were excluded. Although the study was of a relatively large size and long duration, it may not be sufficient to detect possible rare adverse events or long-term risks.

\section{Conclusions}

Galcanezumab treatment was associated with a statistically significant and clinically meaningful reduction in monthly MHDs at doses of 120 and $240 \mathrm{mg}$ given subcutaneously once monthly. Moreover, patients treated with either dose of galcanezumab reported a reduction in the migraine-related impairment 
of function and a significant reduction in migraine-related disability, while also reducing the use of acute migraine medications. Galacanezumab treatment was safe and well tolerated. No clinically meaningful differences in either efficacy or safety were found between the two galcanezumab dose regimens. The results of this investigation confirm findings from several previously published migraine prevention studies of galcanezumab.

\section{Conflict of Interest Statements}

MSM serves on the advisory board for Allergan, Autonomic Technologies Inc, St Jude Medical and Medtronic, and has received payment for the development of educational presentations from Allergan, Autonomic Technologies Inc, Medtronic and electroCore. BKK received lecture fees from Allergan, Sandos, YuYu pharm and SK pharm. VS, BAM, and JYY are full time employees and minor stock holders of Eli Lilly and Company. MHO is a full time employee of Syneos Health.

\section{Funding}

The trial was sponsored by Eli Lilly and Company.

\section{Acknowledgments}

Most importantly, the authors thank the patients who participated in the study, and the following investigators and their respective institutions: William D Koltun, MD, Medical Center for Clinical Research; Marigene D Salazar Sharma, MD, Albuquerque Clinical Trials; Michael Ament, MD, MBA, IMMUNOe International Research Centers; Itay D Melamed, MD, IMMUNOe International Research Centers; Eugene Andruczyk, DO, Clinical Research of Philadelphia; Lesley M Arnold, MD, Univ of Cincinnati College of Medicine; Thomas V Ballard, MD, Mountain View Clinical Research, Inc; William D Byars, MD, Mountain View Clinical Research, Inc; Mushtque Chachar, MD, Novex Clinical Research; Mohammad Munir, MD, Novex Clinical Research; Herman J Downey, MD, Westside Center for Clinical Research; Michael J Downing, MD, FutureSearch Trials; Alexander P Feoktistov, MD, PhD, Diamond Headache Clinic; Chester L Fisher, Jr., MD, MPH, Health Research of Hampton Roads Inc; Kristi K 
George, MD, Josephson Wallack Munshower Neurology; Ira D Glick, MD, Pacific Research Partners, LLC; Dan C Henry, MD, Foothill Family Clinic; Jose C Rafecas, MD, Neurology \& Neuroscience Associates, Inc.; Cynthia L Huffman, MD, Meridien Research; Lissette Jimenez, MD, SomniCare Sleep Institute; Nandita M Joshi, MD, Clinical Neuroscience Solutions Inc; Shawn K Hassler, MD, Optimus Medical Group; Timothy M Koehler, DO, Heartland Research Associates; Rise A Futterer, MD, MPH, Dean Foundation for Health Research and Education; Amelito B Malapira, MD, Sentara Neurology Specialists; Mia Moon, MD, Catalina Research Institute, LLC; Gilbert J Martinez, MD, Catalina Research Institute, LLC; Braden T Nago, MD, POLYCLINIC; Margarita Nunez, MD, High Point Clinical Trials Center; Ashok K Patel, MD, Bio Behavioral Health; Larry D Reed, MD, PhD, Healthcare Research Network - Hazelwood; Alan J Reichman, MD, Clinical Trial Network; Barry J Riskin, MD, Christie Clinic, LLC; Aamer Habib, MD, Christie Clinic, LLC; Christian E Schenk, MD, Cortex, PSC; Jose M Itzcovich-Schuster, MD, Schuster Medical Research Institute; Cynthia B Strout, MD, Coastal Carolina Research Center, Inc.; Duncan Sze-Tu, MD, Regional Clinical Research; Louise A Taber, MD, Arizona Research Center; Louise M Thurman, MD, MPH, IPS Research Company; Joshua A Tobin, MD, Xenoscience; Nicholas G Vatakis, MD, SPRI Clinical Trials, LLC.; Robert A Riesenberg, MD, Atlanta Center of Medical Research; Arifulla Khan, MD, Northwest Clinical Research Center; Steven B GraffRadford, DDS, Cedars Sinai Medical Center; Alan C Newman, DDS, Cedars Sinai Medical Center; Jeffrey B Rosen, MD, Clinical Research of South Florida; Jeffrey White, MD, Urgent Care Specialists, LLC; Narinder Saini, MD, Urgent Care Specialists, LLC; Steve H Choi, MD, Urgent Care Specialists, LLC; JienSup Kim, MD, Axiom Research; Abraham J Nagy, MD, Nevada Headache Institute; Kelly N Taylor, MD, Sensible Healthcare; Thomas G Ledbetter, MD, ClinPoint Trials, LLC; Katie A Julien, MD, Jordan River Family Medicine; Harvey D Schwartz, MD, Sunrise Clinical Research; Fayyaz Ahmed, MD, Hull Royal Infirmary; Brendan Davies, MD, Royal Stoke University Hospital; Peter Goadsby, MD, PhD, Kings College Hospital; Alok Tyagi, MD, Queen Elizabeth University Hospital; Nicholas Silver, MD, PhD, Walton Centre for Neurology and Neurosurgery; Emile Couturier, MD, Boerhaave Medisch Centrum; Wim Mulleners, MD, Canisius-Wilhelmina Ziekenhuis; Michel Ferrari, MD, PhD, Leids 
Universitair Medisch Centrum; Veronique Triebels, MD, Zuyderland Medisch Centrum; Peter van den Berg, MD, PhD, Isala Klinieken; Guus Schoonman, MD, PhD, TweeSteden Ziekenhuis; Angel Luis Guerrero Peral, MD, Hospital Universitario de Valladolid; Jose Miguel Lainez Andres, MD, PhD, Hospital Clínico Universitario de Valencia; Agustin Oterino Duran, MD, Hospital Universitario Marques De Valdecilla; Patricia Pozo Rosich, MD, PhD, Hospital Universitari Vall d'Hebron; Vicente Medrano Martinez, MD, Hospital General De Elda; Joan Prat Rojo, MD, Hospital Universitari de Bellvitge; Michal Bajacek, MD, PhD, Neurologicka ordinace; David Dolezil, MD, PhD, MBA, DADO MEDICAL, s.r.o.; Fabienne Kosova, MD, Clintrial, s.r.o.; Yuliya Rizova, MD, Clintrial, s.r.o.; Hynek Lachmann, MD, Axon Clinical, s.r.o.; Irena Novotna, MD, Fakultni Nemocnice U svate Anny; Klaudia VodickovaBorzova, MD, Brain-Soultherapy s.r.o; Andreas Böger, MD, DRK-Kliniken Nordhessen; Matthias Böhringer, MD, NeuroZentrum Bielefeld; Stefan Braune, MD, Neurozentrum Prien; Tobias Freilinger, MD, Klinikum der Eberhard-Karls-Universität Tübingen; Charly Gaul, MD, Migräne- und Kopfschmerzklinik GmbH \& Co. KG; Heike Israel-Willner, MD, MBA, Charité Universitätsmedizin Berlin; Torsten Kraya, MD, Martin-Luther-Univ Halle-Wittenberg; Arne May, MD, PhD, Universitätsklinikum Hamburg - Eppendorf; Jörg Peltz, MD, PhD, Neuropsychiatrisches Facharztzentrum; Kasja Solbach, MD, Universitätsklinikum Essen; Dagny Holle-Lee, MD, Universitätsklinikum Essen; Peter Storch, MD, Klinikum der Friedrich-Schiller-Universität Jena; Philipp Stude, MD, Praxis Dr. Philipp Stude; Conrado J Estol, MD, PhD, Stat Research; Bibiana B Saravia, MD, Mautalen Salud e Investigacion-Centro de Osteopatías Médicas; Gustavo Fischbein, MD, Instituto de Investigaciones Metabolicas; Osvaldo CJ Bruera, MD, Instituto de Investigaciones Metabolicas; Fernanda M Paez, MD, Aprillus Asistencia e Investigacion - Servicio de neurologia; Yaron River, MD, Hillel Yaffe Medical Center; David Yarnitsky, MD, Rambam Medical Center; Yelena Feingold, MD, Chaim Sheba Medical Center; Gabriel Vainstein, MD, Maccabi Healthcare Services, Kfar Saba; Soo-Jin Cho, MD, PhD, Hallym University Dongtan Sacred Heart Hospital; Chin-Sang Chung, MD, PhD, Samsung Medical Center; Byung Kun Kim, MD, PhD, Eulji General Hospital; Man Ho Kim, MD, PhD, Seoul National University Hospital; Kyungmi Oh, MD, Korea University Guro Hospital; Heui Soo Moon, MD, PhD, 
Kangbuk Samsung Hosp; Min Kyung Chu, MD, PhD, Hallym Univ. of Medicine, Kangnam Sacred Heart Hospital; Kao-Chang Lin, MD, Chi-Mei Medical Center - Yung Kang; Shiang-Ru Lu, MD, Kaohsiung Medical University Chung-Ho Memorial Hospital; Po-Jen Wang, MD, Sin-Lau Hospital; Shuu-Jiun Wang, MD, Taipei Veterans General Hospital; Tzu-Hsien Lai, MD, Far Eastern Memorial Hospital; Freddy G Castro Farfan, MD, Grupo Médico Camino SC; Jose A Gien Lopez, MD, Medical Care and Research, S.A. de C.V.; Elmer G Lopez Meza, MD, Neurociencias Estudios Clinicos; Jose H Nicolini Sanchez, MD, PhD, Grupo Medico Carracci; Sarug Reyes Morales, MD, Instituto Biomedico de Investigacion AC. The authors also thank Sarah Becker-Marrero of Syneos Health for help in editing and proofreading the manuscript. 


\section{Clinical Implications}

- Galcanezumab is safe and efficacious in preventing migraine.

- Galcanezumab reduces migraine-related disability and impairment in functioning.

- The study completion rates of the galcanezumab-treated groups were higher than that of placebo. 
Table 1.

\begin{tabular}{|c|c|c|c|}
\hline Category & $\begin{array}{l}\text { Placebo } \\
\mathrm{N}=461\end{array}$ & $\begin{array}{c}\text { Galcanezumab } 120 \\
\text { mg } \\
\mathrm{N}=\mathbf{2 3 1}\end{array}$ & $\begin{array}{c}\text { Galcanezumab } \\
240 \mathrm{mg} \\
\mathrm{N}=\mathbf{2 2 3}\end{array}$ \\
\hline \multicolumn{4}{|l|}{ Patient Demographics } \\
\hline Age, years, mean (SD) & $42.3(11.3)$ & $40.9(11.2)$ & $41.9(10.8)$ \\
\hline Gender (female), \% & 85.3 & 85.3 & 85.7 \\
\hline Race (white), \% & 70.5 & 71.9 & 68.2 \\
\hline \multicolumn{4}{|l|}{ Disease Characteristics at Baseline } \\
\hline MHD per month, ${ }^{\dagger}$ mean (SD) & $9.2(3.0)$ & $9.07(2.9)$ & $9.06(2.9)$ \\
\hline $\begin{array}{l}\text { MHD with acute medication use per } \\
\text { month,* mean (SD) }\end{array}$ & $7.6(3.4)$ & $7.47(3.3)$ & $7.47(3.3)$ \\
\hline MIDAS total score, mean (SD) & $34.3(31.0)$ & $30.9(27.9)$ & $32.8(28.8)$ \\
\hline MSQ RF-R, * mean (SD) & $51.4(15.7)$ & $52.5(14.8)$ & $51.7(16.3)$ \\
\hline PGI-S, * mean $(\mathrm{SD})$ & $4.3(1.2)$ & $4.1(1.2)$ & $4.2(1.2)$ \\
\hline $\begin{array}{l}\text { Duration of migraine illness, years, } \\
\text { mean (SD) }\end{array}$ & $21.2(12.8)$ & $19.93(11.7)$ & $20.01(12.1)$ \\
\hline Migraine attacks per month, mean (SD) & $5.7(1.8)$ & $5.54(1.8)$ & $5.66(1.8)$ \\
\hline Number of headache days, mean (SD) & $10.7(3.5)$ & $10.56(3.4$ & $10.74(3.7)$ \\
\hline MHD category $\geq 8, \%$ & 66.6 & 66.7 & 67.7 \\
\hline Prior preventive treatment, $\%$ & 64.6 & 68.0 & 64.6 \\
\hline $\begin{array}{l}\geq 2 \text { failed preventive treatments mean } \\
\text { (SD) }\end{array}$ & $63(13.7)$ & $34(14.7)$ & $34(15.3)$ \\
\hline \multicolumn{4}{|l|}{ Region } \\
\hline North America ${ }^{a}, \%$ & 48.6 & 48.5 & 49.3 \\
\hline Europe, $\%$ & 26.5 & 26.0 & 26.5 \\
\hline Other, \% & 25.0 & 25.5 & 24.2 \\
\hline
\end{tabular}

${ }^{\dagger}$ Primary and *key secondary outcome measures. Other characteristics may be associated with non-key secondary measures and (except for MIDAS) fall outside the scope of this manuscript. They will be

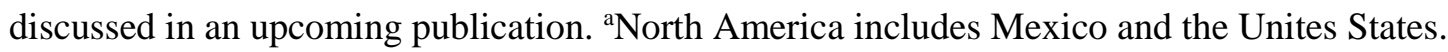

Abbreviations: MHD=migraine headache day; MIDAS=Migraine Disability Assessment; MSQ RFR=Migraine-Specific Quality of Life Questionnaire Role Function-Restrictive; N=the intent-to-treat population for each dosing regimen; PGI-S=Patient Global Impression of Severity; SD=standard deviation. 
Table 2. Primary Objectives, Key Secondary Objectives, and the MIDAS

\begin{tabular}{|c|c|c|c|c|c|c|c|}
\hline \multirow[t]{2}{*}{ Outcome measures } & \multirow{2}{*}{$\begin{array}{c}\text { Placebo } \\
\text { N=461 } \\
\text { LSM (SE) } \\
(95 \% \mathrm{CI})\end{array}$} & \multicolumn{2}{|c|}{$\begin{array}{c}\text { Galcanezumab } \\
\begin{array}{c}120 \mathrm{mg} \\
\mathrm{N}=231\end{array}\end{array}$} & \multicolumn{2}{|c|}{$\begin{array}{c}\text { Galcanezumab } \\
\mathbf{2 4 0} \mathbf{~ m g} \\
\mathrm{N}=\mathbf{2 2 3}\end{array}$} & \multicolumn{2}{|l|}{ Adjusted } \\
\hline & & $\begin{array}{l}\mathrm{LSM}(\mathrm{SE}) \\
(95 \% \mathrm{CI})\end{array}$ & $\mathrm{p}$-value & $\begin{array}{l}\text { LSM (SE) } \\
(95 \% \mathrm{CI}) \\
\end{array}$ & $\mathrm{p}$-value & $\begin{array}{c}\text { significance } \\
\text { level }\end{array}$ & Sig. \\
\hline Overall change in MHD & $\begin{array}{l}-2.3(0.2) \\
(-2.7,-1.9)\end{array}$ & $\begin{array}{l}-4.3(0.3) \\
(-4.8,-3.8)\end{array}$ & $<0.001$ & $\begin{array}{l}-4.2(0.3) \\
(-4.7,-3.7)\end{array}$ & $<0.001$ & 0.026 & $\mathrm{~S}$ \\
\hline \multicolumn{8}{|l|}{$\begin{array}{c}\text { Mean proportion (as \%) } \\
\text { of responders }\end{array}$} \\
\hline$\geq 50 \%$ & $\begin{array}{l}36(1.7) \\
(33,39)\end{array}$ & $\begin{array}{l}59.3(2.4) \\
(55,64)\end{array}$ & $<0.001$ & $\begin{array}{l}56.5(2.5) \\
(52,61)\end{array}$ & $<0.001$ & 0.025 & S \\
\hline$\geq 75 \%$ & $\begin{array}{c}17.8(1.3) \\
(15,21)\end{array}$ & $\begin{array}{c}33.5(2.3) \\
(29,38)\end{array}$ & $<0.001$ & $\begin{array}{c}34.3(2.3) \\
(30,39)\end{array}$ & $<0.001$ & 0.025 & $\mathrm{~S}$ \\
\hline $100 \%$ & $\begin{array}{l}5.7(0.7) \\
(4.4,7.3)\end{array}$ & $\begin{array}{c}11.5(1.4) \\
(9,15)\end{array}$ & $<0.001$ & $\begin{array}{c}13.8(1.5) \\
(11,17)\end{array}$ & $<0.001$ & 0.025 & S \\
\hline $\begin{array}{l}\text { Change in MHD with } \\
\text { acute migraine } \\
\text { medication use }\end{array}$ & $\begin{array}{l}-1.9(0.2) \\
(-2.2,-1.5)\end{array}$ & $\begin{array}{c}-3.7(0.2) \\
(-4.1,-3.2)\end{array}$ & $<0.001$ & $\begin{array}{l}-3.6(0.2) \\
(-4.1,-3.2)\end{array}$ & $<0.001$ & 0.0125 & $\mathrm{~S}$ \\
\hline MSQ RF-R & $\begin{array}{l}19.7(0.9) \\
(17.9,21.5)\end{array}$ & $\begin{array}{l}28.5(1.2) \\
(26.2,30.7)\end{array}$ & $<0.001$ & $\begin{array}{c}27(1.2) \\
(24.7,29.3)\end{array}$ & $<0.001$ & 0.025 & $\mathrm{~S}$ \\
\hline MIDAS total score & $\begin{array}{l}-12(1.3) \\
(-14.5,-9.5)\end{array}$ & $\begin{array}{c}-21.2(1.6) \\
(-24.3,-18.1)\end{array}$ & $<0.001$ & $\begin{array}{c}-20.2(1.6) \\
(-23.4,-17.1)\end{array}$ & $<0.001$ & N/A & N/A \\
\hline PGI-S & $\begin{array}{l}-0.9(0.1) \\
(-1.1,-0.8)\end{array}$ & $\begin{array}{l}-1.2(0.1) \\
(-1.4,-1.1)\end{array}$ & 0.002 & $\begin{array}{l}-1.2(0.1) \\
(-1.3,-1.0)\end{array}$ & 0.012 & 0.025 & $S$ \\
\hline
\end{tabular}

Data are presented as the change from baseline. P-values are the given versus placebo. The adjusted significance level represents the significance threshold adjusted for multiplicity. If $p$-value is less than or equal to the adjusted significance level, then the results are statistically significant after adjustment for multiplicity. MIDAS was not a key secondary outcome measure, and was not subjected to multiplicity adjustment.

Abbreviations: $\mathrm{CI}=$ Confidence intervals; $\mathrm{LSM}=$ least squares mean; $\mathrm{MHD}=$ migraine headache day; MIDAS=Migraine Disability Assessment; MSQ RF-R=Migraine-Specific Quality of Life Questionnaire Role Function-Restrictive; N=the intent-to-treat population for each dosing regimen; N/A=Not applicable; PGI-S=Patient Global Impression of Severity; PL=placebo; S=Significant; SE=standard error, Sig.=significance. 
Table 3. Overview of Adverse Events

\begin{tabular}{lccccc}
\hline & Placebo & & & Galcanezumab \\
Category & N=461 & $\begin{array}{c}\mathbf{1 2 0} \mathbf{~ m g} \\
\mathbf{N = 2 2 6} \\
\mathbf{n}(\boldsymbol{\%})\end{array}$ & p-value & $\begin{array}{c}\mathbf{N = 2 2 8} \\
\mathbf{n}(\boldsymbol{\%})\end{array}$ & p-value \\
\hline TEAEs & $287(62.3)$ & $147(65.0)$ & 0.501 & $163(71.5)$ & 0.017 \\
Serious AEs & $5(1.1)$ & $5(2.2)$ & 0.31 & $7(3.1)$ & 0.07 \\
Deaths & $0(0.0)$ & $0(0.0)$ & & $0(0.0)$ & \\
$\begin{array}{l}\text { Discontinuation due to } \\
\quad \text { AEs }\end{array}$ & $8(1.7)$ & $5(2.2)$ & 0.767 & $9(4.0)$ & 0.114 \\
\hline
\end{tabular}

P-values vs. placebo. No p-values were computed for "Deaths", since all values were 0.Abbreviations: AEs=adverse events; $\mathrm{N}=$ the safety population for each dosing regimen; TEAEs=treatment-emergent adverse events. 
Table 4. Treatment-Emergent Adverse Events $\geq 2 \%$ Galcanezumab Total

\begin{tabular}{|c|c|c|c|c|c|}
\hline Category & $\begin{array}{c}\text { Placebo } \\
\mathrm{N}=461 \\
\mathrm{n}(\%)\end{array}$ & $\begin{array}{c}\text { Galcanezumab } \\
\begin{array}{c}120 \mathrm{mg} \\
\mathrm{N}=226 \\
\text { n }(\%)\end{array} \\
\end{array}$ & p-value & $\begin{array}{c}\text { Galcanezumab } 240 \\
\text { mg } \\
\mathrm{N}=\mathbf{2 2 8} \\
\mathrm{n}(\%) \\
\end{array}$ & p-value \\
\hline $\begin{array}{l}\text { Subjects with } \geq 1 \\
\text { TEAE }\end{array}$ & $287(62.3)$ & $147(65.0)$ & .004 & $163(71.5)$ & $<.001$ \\
\hline Injection site pain & $39(8.5)$ & $21(9.3)$ & .774 & $20(8.8)$ & .89 \\
\hline $\begin{array}{l}\text { Injection site } \\
\text { reaction }\end{array}$ & $0(0.0)$ & $7(3.1)$ & $<.001$ & $18(7.9)$ & $<.001$ \\
\hline $\begin{array}{l}\text { Injection site } \\
\text { erythema }\end{array}$ & $4(0.9)$ & $6(2.7)$ & .089 & $7(3.1)$ & .048 \\
\hline $\begin{array}{l}\text { Injection site } \\
\text { pruritus }\end{array}$ & $0(0.0)$ & $6(2.7)$ & .001 & $7(3.1)$ & $<.001$ \\
\hline $\begin{array}{l}\text { Injection site } \\
\text { swelling }\end{array}$ & $0(0)$ & $5(2.2)$ & .004 & $1(0.4)$ & .331 \\
\hline Nasopharyngitis & $41(8.9)$ & $19(8.4)$ & .886 & $16(7.0)$ & .464 \\
\hline $\begin{array}{l}\text { Upper respiratory } \\
\text { tract infection }\end{array}$ & $16(3.5)$ & $13(5.8)$ & .164 & $12(5.3)$ & .306 \\
\hline Dizziness & $10(2.2)$ & $8(3.5)$ & .314 & $7(3.1)$ & .448 \\
\hline Influenza & $14(3.0)$ & $3(1.3)$ & .203 & $10(4.4)$ & .381 \\
\hline Fatigue & $12(2.6)$ & $6(2.7)$ & 1.000 & $5(2.2)$ & 1.000 \\
\hline Diarrhea & $11(2.4)$ & $7(3.1)$ & .615 & $3(1.3)$ & .407 \\
\hline
\end{tabular}

P-values vs. placebo.Abbreviations: $\mathrm{N}=$ the safety population for each dosing regimen; TEAE=treatmentemergent adverse event.

Figure 1. Study Protocol. a Patients randomized to the galcanezumab $120 \mathrm{mg}$ dose received a loading dose of $240 \mathrm{mg}$ at the first injection only (Visit 3). Abbreviation: SP=Study Period; X indicates visits when dosing occurred. 
Figure 2. For the superchain multiple testing procedure, Dunnett's test was used to test the primary null hypotheses. When at least one of the 2 primary null hypotheses was rejected, the Hochberg procedure was to be used to test the $50 \%$ and $75 \%$ response rate endpoints at the corresponding dose(s). Upon rejection of these null hypotheses, the Bonferroni-Holm procedure was to be used to test the change in the use of acute migraine treatment and the change in functioning for the MSQ, at the corresponding doses. Following rejection of these null hypotheses, the hypotheses of $100 \%$ response rate and PGI-S were tested sequentially at the corresponding doses. When all null hypotheses for a single dose were rejected, available alpha was to be propagated to the other dose for continued testing of available nonrejected null hypotheses. In the diagram, propagation weights are denoted along edges connecting boxes, and families of hypotheses are shown in the boxes.

Abbreviations: acute meds=MHD with the use of acute treatment; $\mathrm{MHD}=$ the number of monthly migraine headache days (mean change from baseline); MSQ RF-R=Migraine-Specific Quality of Life

Questionnaire Role Function-Restrictive; PGI-S=Patient Global Impression-Severity; RR=response rate in MHD.

Figure 3. CONSORT diagram showing patient disposition throughout the trial. *p<.05 (vs. placebo); $\dagger \mathrm{p}<.05$ (vs. galcanezumab $120 \mathrm{mg}$ ).

Figure 4a. The overall LS mean change from Months 1 through 6 in monthly migraine headache days. The inset table shows the LS mean change from placebo for both doses of galcanezumab. Figure 4b. The LS mean change at each of the Months 1 through 6 in monthly migraine headache days. ***p<.001 (vs. placebo).

Abbreviations: LS=least squares; $\mathrm{SE}=$ standard error.

Figure 5. The mean percent of patients with $\geq 50 \%, \geq 75 \%$, and $100 \%$ reductions in monthly migraine headache days. $* * * \mathrm{p}<0.001$ (vs. placebo).

Abbreviation: $\mathrm{SE}=$ standard error.

Figure 6. The LS mean change in migraine headache days with the use of acute migraine medications. $* * * \mathrm{p}<0.001$ (vs. placebo).

Abbreviations: LS=least squares; $\mathrm{SE}=$ standard error.

Figure 7. The LS mean change in the Migraine-Specific Quality of Life Questionnaire Role FunctionRestrictive domain. $* * * \mathrm{p}<0.001$ (vs. placebo).

Abbreviations: LS=least squares; $\mathrm{SE}=$ standard error.

Figure 8. The LS mean change in the Patient Global Impression-Severity rating. **p<0.01; ${ }^{*} \mathrm{p}<0.05$ (vs. placebo).

Abbreviations: LS=least squares; $\mathrm{SE}=$ standard error.

Figure 9. The LS mean change in the Migraine Disability Assessment score. ${ }^{* * *} \mathrm{p}<0.001$ (vs. placebo).

Abbreviations: $\mathrm{LS}=$ least squares; $\mathrm{SE}=$ standard error. 



\section{References}

1 Steiner TJ, Stovner LJ, Birbeck GL. Migraine: the seventh disabler. Cephalalgia 2013; 33: 289290.

2 GBD 2016 Neurological Disorders Collaborator Group. Global, regional, and national incidence, prevalence, and years lived with disability for 328 diseases and injuries for 195 countries, 19902016: a systematic analysis for the Global Burden of Disease Study 2016. Lancet 2017; 390: $1211-$ 2159.

3 The International Classification of Headache Disorders, 3rd edition (beta version). Cephalalgia 2013; 33: 629-808.

4 Lipton RB, Silberstein SD. Episodic and chronic migraine headache: breaking down barriers to optimal treatment and prevention. Headache 2015; 55(Suppl 2): 103-122.

5 Peck KR, Johnson YL, Smitherman TA. Migraine. Handb Clin Neurol 2016; 138: 283-293.

6 Lipton RB, Bigal ME, Diamond M, et al. Migraine prevalence, disease burden, and the need for preventive therapy. Neurology 2007; 68: 343-349.

7 Berger A, Bloudek LM, Varon SF, et al. Adherence with migraine prophylaxis in clinical practice. Pain Pract 2012; 12: 541-9.

8 Diamond S, Bigal ME, Silberstein S, et al. Patterns of diagnosis and acute and preventive treatment for migraine in the United States: results from the American Migraine Prevalence and Prevention study. Headache 2007; 47: 355-363.

9 Loder EW, Rizzoli P. Tolerance and loss of beneficial effect during migraine prophylaxis: clinical considerations. Headache 2011; 51: 1336-1345.

10 Goadsby PJ, Edvinsson L, Ekman R. Release of vasoactive peptides in the extracerebral circulation of humans and the cat during activation of the trigeminovascular system. Ann Neurol 1988; 23: 193-196.

11 Goadsby PJ, Edvinsson L, Ekman R. Vasoactive peptide release in the extracerebral circulation of humans during migraine headache. Ann Neurol 1990; 28: 183-187.

12 Goadsby PJ, Holland PR, Martins-Oliveira M, et al. Pathophysiology of migraine: a disorder of sensory processing. Physiol Rev 2017; 97: 553-622.

13 Burstein R, Noseda R, Borsook D. Migraine: multiple processes, complex pathophysiology. $J$ Neurosci 2015; 35: 6619-6629.

14 Iyengar S, Ossipov MH, Johnson KW. The role of calcitonin gene-related peptide in peripheral and central pain mechanisms including migraine. Pain 2017; 158: 543-559.

15 Lassen LH, Jacobsen VB, Pedersen PA, et al. Human calcitonin gene-related peptide (hCGRP)induced headache in migraineurs. Eur J Neurol 1998; 5(Supplement 3): S63.

16 Lassen LH, Haderslev PA, Jacobsen VB, et al. CGRP may play a causative role in migraine. Cephalalgia 2002; 22: 54-61. 
17 Goadsby PJ, Edvinsson L. The trigeminovascular system and migraine: studies characterizing cerebrovascular and neuropeptide changes seen in humans and cats. Ann Neurol 1993; 33: 48-56.

18 Dodick DW, Goadsby PJ, Spierings ELH, et al. Safety and efficacy of LY2951742, a monoclonal antibody to calcitonin gene-related peptide, for the prevention of migraine: a phase 2, randomised, double-blind, placebo-controlled study. Lancet Neurol 2014; 13: 885-892.

19 Skljarevski V, Oakes TM, Zhang Q, et al. Effect of different doses of galcanezumab vs placebo for episodic migraine prevention: a randomized clinical trial [Epub ahead of print]. JAMA Neurol 2017 Dec 18. doi: 10.1001/jamaneurol.2017.3859.

20 Silberstein SD, Holland S, Freitag F, et al. Evidence-based guideline update: pharmacologic treatment for episodic migraine prevention in adults: report of the Quality Standards Subcommittee of the American Academy of Neurology and the American Headache Society. Neurology 2012; 78 : $1337-1345$.

21 Kordzakhia G, Dmitrienko A. Superchain procedures in clinical trials with multiple objectives. Stat Med 2013; 32: 486-508.

22 Goadsby PJ, Sprenger T. Current practice and future directions in the prevention and acute management of migraine. Lancet Neurol 2010; 9: 285-298.

23 Stauffer VL, Dodick DW, Zhang Q, et al. Evaluation of galcanezumab for the prevention of episodic migraine: results from the EVOLVE-1 study, a randomized phase 3 placebo-controlled clinical trial. Submitted to JAMA Neurol 2018. 